\title{
Bioactive glass as a treatment option for dentin hypersensitivity
}

\author{
Luiza Pereira Dias da Cruz, ${ }^{1}$ Inger Teixeira de Campos Tuñas ${ }^{1}$ \\ ${ }^{1}$ Department of Social and Preventive Dentistry, School of Dentistry, Rio de Janeiro Federal University, Rio de Janeiro, RJ, Brazil \\ - Conflicts of interest: none declared.
}

\section{ABstract}

Objective: analyze significant information available in the literature regarding $\mathrm{DH}$ and its treatment options with a focus on the role of bioactive glass-based toothpastes. Material and Methods: we conducted a literature search of electronic databases, including PUBMed and Scielo, by using the key words: (Dentifrice OR Dentifrices) OR (Toothpaste OR Toothpastes) AND (Treatment) AND (Dentin OR Dentine OR Tooth) AND (Hypersensitivity OR Sensitivity) AND (Dentifrício OR Dentifrícios) AND (Tratamento OR Tratamentos) AND (Dentinária OR Dentina OR Dente) AND (Hipersensibilidade OR Sensibilidade). The papers found were analysed regarding title and abstract contents in order to eliminate the ones that were out of context and not relevant to the review. Results: after this first filter, 31 papers were selected, in which the full text available was considered of good quality and relevant to the context. The languages of the papers were predominantly English and Portuguese and articles published before 2002 were excluded. The use of bioactive glasses in toothpaste formulations has been advocated as a possible solution to managing DH. Conclusion: there are several different options for treating $\mathrm{DH}$, although dentifrices containing bioactive glass are a promising option.

Keywords: Dentine hypersensitivity; Diagnostic; Treatment; Bioglass.

\section{Introduction}

$\mathrm{D}$ entine hypersensitivity $(\mathrm{DH})$ is a population-wide problem, that affects approximately a quarter of the adult population and directly influences an individual's quality of life. The prevalence distribution of $\mathrm{DH}$ is related to social and dietary aspects among different populations. Epidemiology analyses usually include patient questionnaires or clinical examinations, and most articles have stated that the prevalence for $\mathrm{DH}$ is between $3 \%$ and $57 \%$. Apparently, all age groups are affected, but there is a considerable peak between 30 and 40 years. Women have a higher frequency of hypersensitivity than men. Intraorally, $\mathrm{DH}$ occurs more often in exposed buccal dentine in permanent teeth. ${ }^{1-3}$

The causes of DH have been historically unclear, and the etiological factors related to dental sensitivity are still being studied. It is known that non-carious loss of cervical tooth structure is related to specific factors. ${ }^{4,5}$ In normal healthy teeth, it is possible for pain to arise from exposed dentine at the cervix or root. The loss of enamel or periodontal and cemental tissues, makes the dentine more susceptible to physio-chemical attack. ${ }^{5}$

$\mathrm{DH}$ has been defined as an acute pain that originates from exposed dentine in response to thermal, evaporative, tactile, osmotic, or chemical stimuli that cannot be ascribed to any other disease strategies. Therefore, changes in the oral environment, including temperature, pressure, solute concentrations, and local charges, may change the fluid flow in the dentinal tubules which causes the symptoms of pain. ${ }^{6}$

The hydrodynamic theory explaining the mechanism of DH involves the idea that stimuli applied to the exposed dentine can increase the dentine tubule's fluid flow and trigger a mechanoreceptor response. According to the hydrodynamic theory, there are two methods for treating DH: 1) coating the dentine surface and blocking the tubules or 2) desensitizing the pulpal nerves that are responsible for pain. ${ }^{6}$

Different products such as fluorides, oxalates, varnished, adhesive resins, sealants, lasers, Portland cement and Bioglass, have been tested for blocking open dentinal tubules on the root surface. Most current research is focused on reduction of dentine permeability via tubule occlusion as an important strategy for treating sensitive teeth. Nevertheless, reducing dentine permeability can also prevent activation of intradental nerves. ${ }^{4,6}$

The aim of this study, therefore, was to review the published literature on treatment options for $\mathrm{DH}$, among overthe-counter (OTC) and professionally-applied (in-office) products available, with a focus on the role of bioactive glass-based toothpastes. We discuss the best options currently available to clinicians.

\section{Material and Methods}

A literature search of electronic databases, including PUBMed and Scielo, with the key terms of (Dentifrice OR Dentifrices) OR (Toothpaste OR Toothpastes) OR (Treatment) AND (Dentin OR Dentine OR Tooth) AND (Hypersensitivity OR Sensitivity) AND (Dentifrício OR Dentifrícios) AND (Tratamento OR Tratamentos) AND (Dentinária OR Dentina OR Dente) AND (Hipersensibilidade OR Sensibilidade). The papers found were analysed regarding title and abstract contents in order to eliminate the ones that were out of context and not relevant to the review. Also, 31 papers were selected after this first filter, in which the full text available was considered of good quality and relevant to the context (Figure 1). The languages of the papers were predominantly English and Portuguese. The 
publication time was limited to the 15 -year period through December 2016, with a focus on the latest articles.

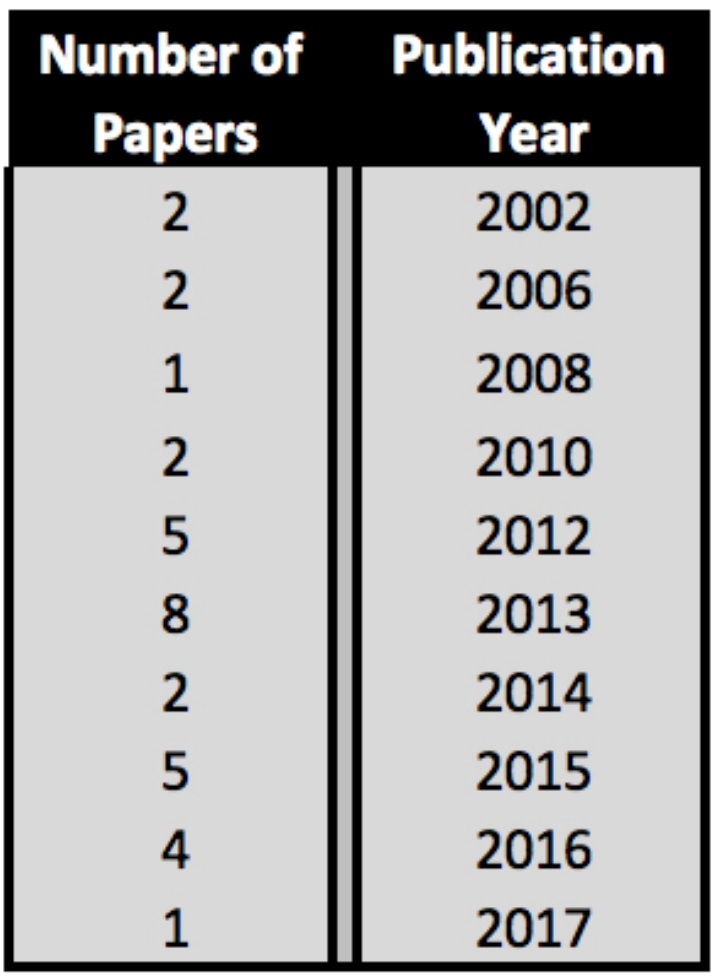

Figure 1. Number of papers per publication year

\section{Results Literature Review}

\section{Dentine Hypersensitivity}

There are many theories to explain the mechanism of $\mathrm{DH}$ : direct innervation theory, odontoblast receptor theory, or hydrodynamic theory. The most widely accepted is the hydrodynamic theory proposed by Gysi in the nineteenth century. Some years later, Brännström et al. published several studies confirming this theory. Brännström's theory which was postulated in 1981, states that exposed dentine is vulnerable to many stimuli, thus these stimuli applied to exposed dentine cause an increase in fluid flow in the dentinal tubules that can trigger a mechanoreceptor response in A-beta and A-delta pulpal nerve fiberes. ${ }^{3,4,6,7}$

However, occlusion of dentinal tubules can cause a decrease in the diameter of the dentinal tubules which reduces fluid flow. The pain caused by exposed cervical dentine is related to the density of the tubules. The patency of the tubules is relevant, and dentine with high conductance has low resistance and vice-versa. Moreover, scanning electron microscopy (SEM) studies have shown that the number of tubules in sensitive dentine is eight times higher than in non-sensitive dentine. ${ }^{1,7-10}$

The smear layer, which is a layer of organic and inorganic material created or removed when tooth brushing, can seal the dentinal tubules inconsistently; similarly, removal of the smear layer increases dentine's hydraulic conductance. On the other hand, brushing in the presence of dietary acids can remove the smear layer., ${ }^{4,8,11}$

Tooth sensitivity pain is mainly caused by a pain-signalling impulse generated by intradental nerves. $\mathrm{DH}$ has the aspects of chronic pain but the sensory aspects of acute pain. Painful stimuli, such as, cavity preparation for restorations, result in an increase in pulpal blood flow, and increases in intrapulpal pressure and outward dentine fluid flow. Inflammation can exacerbate $\mathrm{DH}$ symptoms by making the intradental nerve endings more responsive to dentine fluid flow. When the exposed dentine receives any stimuli, intradental nerves are activated by fluid shifts in the tubules. Evidence for this theory has come from studies that have confirmed the hydrodynamic hypothesis. ${ }^{4,5}$

Nevertheless, there is still ongoing research regarding how exactly dentine sensitivity leads to pain. It is unknown whether nerve endings are directly excited by fluid flow or whether the transduction mechanisms are related to odontoblasts. Studies have demonstrated that it is likely that an inflammatory reaction takes place in the pulp during $\mathrm{DH} .{ }^{1}$

\section{Dentine Hypersensitivity Etiology and Diagnosis}

The exposed cervical dentine that leads to $\mathrm{DH}$ can be a consequence of various problems; for instance, tooth brushing with abrasive toothpaste and/or tool procedures, some periodontal procedures (such as periodontal pocket scaling), post-surgical periodontal disease (recession induced by management), bulimia, xerostomia, high-acid food/ drink consumers, chewing "smokeless" or "snuff" tobacco, whitening procedures, and developmental lesions (rare conditions in which surface morphology of teeth change). ${ }^{1,4,9,12}$ Periodontal therapy is a significant cause of DH. Studies have shown that approximately $84 \%$ of the patients who have undergone periodontal treatments experienced hypersensitivity afterwards. ${ }^{13,14}$

Nevertheless, gingival recession that results in exposure of cervical dentine is the main cause of $\mathrm{DH}$, and there is a clear increase in the number of cases among the geriatric population. ${ }^{15}$

The pathogenesis of $\mathrm{DH}$ is thought to involve lesion localization and lesion initiation. ${ }^{3}$ The localization of the lesion could be gingival recession exposing dentine or loss of hard tissue exposing dentine. Triggers for the lesion to initiate could be abrasion, erosion, plaque, or bleaching. ${ }^{\text {? }}$

All in all, accordingly to West N. et al. factors that can affect susceptibility are "salivary buffering and flow rate, pellicle thickness and charge, movements of soft tissues, distribution and time of acidic liquid in the oral cavity, tooth structure and remineralizing potential". Additionally, the biotype of the gingival margin, oral hygiene, and dietary habits can predispose the individual to the condition.? 
The diagnosis of $\mathrm{DH}$ is based on anamneses and clinical examinations. There are prior conditions that need to be excluded to arrive at a definitive diagnosis of $\mathrm{DH}$, including cracked teeth, dental caries, pulpitis, fractured restorations, medication sensitivity, post-non-surgical/surgical periodontal sensitivity, palatogingival groove and non-odontogenic origins, such as atypical facial pain. ${ }^{1,412}$

The procedures that patients must undergo include extra- and intra-oral examination of tissues, radiographs, and vitality tests. The diagnosis can involve some clinical techniques, such as reconstruction of stimuli by air, water, and sound. Evaporative, cold, and osmotic stimuli increase the fluid flow in the tubules, by using, for example, a triple syringe air jet. Cold is used as a positive stimulus clinically. Additionally, intra-oral examinations include palpitation for periodontal involvement and fiber-optic transillumination for diagnosing tooth fracture. Finally, the clinical data plus the medical history can show whether the pain is from DH or other causes. ${ }^{1,7,12}$

\section{Dentine Hypersensitivity Treatment}

The materials available for $\mathrm{DH}$ treatment range from desensitizing mouthwashes and dentifrices to varnishes and restorative materials. Desensitizing agents are classified on the basis of the mode of administration and mode of action (Figure 2). ${ }^{1,3,16}$

\section{Classification of desensitizing agents by their mode} of administration and mode of action

\begin{tabular}{|c|c||c|}
\hline & $\begin{array}{c}\text { Mode of } \\
\text { administration }\end{array}$ & $\begin{array}{c}\text { At-home desentizing agents } \\
\text { In-office treatments }\end{array}$ \\
\cline { 2 - 3 } $\begin{array}{c}\text { Desensitizing } \\
\text { agents }\end{array}$ & $\begin{array}{c}\text { Mode of } \\
\text { action }\end{array}$ & $\begin{array}{c}\text { Nerve desentization } \\
\text { Protein preciptation } \\
\text { Occlude dentinal tubules } \\
\text { Dentine adhesive sealers }\end{array}$ \\
& & $\begin{array}{c}\text { Lasers } \\
\text { Homeophatic medication }\end{array}$ \\
\hline
\end{tabular}

Figure 2. Classification of desensitizing agents by their mode of administration and mode of action. Adapted from Miglani S, Aggarwal V, Ahuja B. Dentin Hypersensitivity: Recent trends in management. J Conserv Dent. 2010;13(4):218-24

Therefore, the most common types of desensitizing agents that are discussed and clinically relevant currently are the ones that block pulp nerve response (potassium salts) and that occlude dentinal tubules (Figure 3). ${ }^{1,17}$

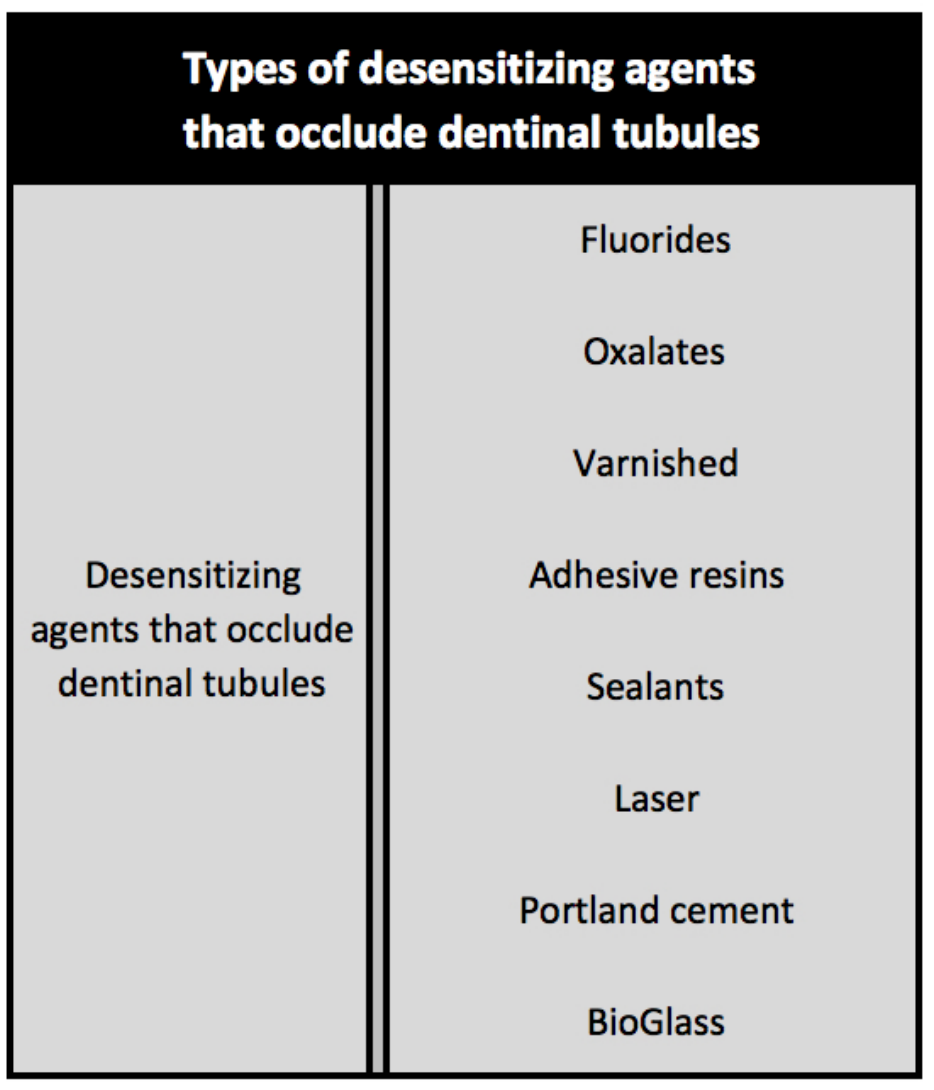

Figure 3. Types of desensitizing agents that occlude dentinal tubules. Adapted from Davari AR, Ataei E, Assarzadeh H. Dentin Hypersensitivity: Etiology, Diagnosis and Treatment. J Dent (Shiraz). 2013;14(3):136-45

An ideal desensitizing agent should have a rapid action with long-term effects, be non-irritating to pulp, painless, easy to apply, and non-staining. Toothpastes are an easy and economical solution for desensitizing in-home treatments. Moreover, they are classified by the characteristics of the ingredients (Figure 4). ${ }^{3,18}$

\section{Summary of substances that occlude detine tubules}

\section{Substance}

\section{Strontium (chloride, acetate) \\ Stannous fluoride \\ Calcium sodium phosphosilicate \\ Oxalates \\ Fluorides \\ Arginine and calcium carbonate \\ Nanoparticles with various functionalizing agents}

Figure 4. Ingredients of dentifrices that are responsible for occluding the dentinal tubules. Adapted from Arnold WH, Prange M, Naumova EA. Effectiveness of various toothpastes on dentine tubule occlusion. J Dent. 2015:43(4):440-449 
Furthermore, there are presently an increasing number of options and techniques to treat $\mathrm{DH}$, which can cause problems with selection of an appropriate treatment. Moreover, patients and even doctors often underestimate the problem. Questionnaire studies have shown that there is a difference between the perceptions of patients who self-diagnose the problem and of clinicians who treat the condition. There is clearly a difficulty in determining the type of pain and whether or not it is related to other problems. The clinical features of $\mathrm{DH}$ are transient and patients do not always present with $\mathrm{DH}$ at examination even though they may suffer from it regularly. For patients, this could lead to stress and emotional problems that directly affect the symptoms, although individuals do not see it as a severe health problem. Furthermore, many studies have concluded that there is a clear lack of awareness among clinicians of the relevance of $\mathrm{DH}$ in patient's quality of life. , $^{42,16}$

It is important to treat not only the pain symptoms but also the underlying dental lesion. A suggested management strategy for treating DH is to first obtain a full detailed clinical and dietary history from the patient. Moreover, differential diagnosis should be performed to exclude other possible conditions. In addition, the professional should manage etiological and predisposing factors that would make the patient susceptible to the condition. If the sensitivity is diagnosed as mild or moderate, then in-home therapy should be prescribed. On the other hand, if the sensitivity is severe or there is no relief after 2-4 weeks of in-home treatment, then, in-office therapy should be considered. ${ }^{3,5}$

For every treatment, it is crucial that the patients are monitored constantly by the clinician to enhance the efficacy of the treatment, while maintaining a strategy-based paradigm. Nevertheless, some current problems remain to be resolved; for instance, under- or overestimation of the condition, difficulty in establishing the correct diagnosis in daily practice, the impact on the patient's quality of life even when the condition is correctly diagnosed and warnings regarding possible side-effects of the treatments. ${ }^{12}$

\section{Bioactive Glasses}

Bioactive materials can be used to replace damaged tissues. In 1969, bioactive glasses were discovered as a second-generation alternative for bone bonding of an implant with a host tissue through chemical reactions. Therefore, their relevance in bone research is very significant because they have the capacity of producing hydroxyapatite, by a chemical bond that produces a surface layer, and inducing osteogenesis in physiological systems. ${ }^{2,6}$

Bioactive glasses have osteoconductive functions and physicochemical properties such as, particle size, composition, porosity, low $\mathrm{pH}$ and solubility, that have been improved in order to gain better biological responses. Because of its $\mathrm{pH}$, is ascribed to bioactive glasses an antimicrobial effect. ${ }^{19}$

Since 1985, 45S5 Bioglass has been used clinically, as a third-generation biomaterial, for tissue reparation using gene activation proprieties. ${ }^{2}$

In dentistry, the application of bioactive glasses is related to bone replacement implants in edentulous patients, to provide a more stable ridge for denture construction. Bioactive glasses were first used and indicated for periodontal diseases and bone defects as a method of reconstruction of bone. Dentine and bone have various similarities; hence, biocompatible glasses can be an efficient material for dentine tubule occlusion. . $^{2,620}$

Bioactive glass is composed of silicon, sodium, calcium, and phosphorus oxides with specific percentages; in addition, it is a source of fluoride and calcium ions that are released when the glass is in contact with saliva..$^{20,21}$

When in contact with biological fluids, such as saliva, bioglass particles react. Three process take place; leaching and formation of silanols, dissolution of the glass network, and precipitation. Precipitation is an important process for occluding dentinal tubules. The formation of a layer composed of calcium and phosphate induced by the release of these ions from the glass can mechanically occlude dentinal tubules and lower the permeability levels of the teeth. This layer is crystallized into hydroxyapatite, and the presence of silica can accelerate the maturation of hydroxyapatite. Basically, bioactive glass triggers an osteoblast cell cycle, which leads to rapid cell proliferations and differentiation. ${ }^{3,16,20,22}$

Toothpastes as a Treatment for Dentine Hypersensitivity Some toothpastes are designed to treat sensitive teeth and may include an analgesic compound. In this case, potassium saline is responsible for maintaining high levels of potassium ion extracellularly, which prevents re-polarization of the nerve cell membrane and inhibits the transmission of impulses. In brief, potassium nitrate acts by blocking neural transmission, which reduces DH symptoms. However, studies are still trying to find substantial evidence on potassium salts in the efficient reduction of DH. Some studies have shown that toothpastes containing calcium sodium phosphosilicate or NovaMin ${ }^{\circledR}$ can block dentinal tubules more effectively than can potassium salts. ${ }^{17,18,22}$

Usually, the dentifrice composition includes fluoride (to protect against caries), an abrasive component (that provides the cleaning ability), substances that inhibit germ growth, and other ingredients, such as a binder, humectant, sweetener, flavor and surfactant. ${ }^{5,18,22}$ Fluoride is a compound that can cause remineralization that can then cause precipitation that can block dentinal tubules and increase dentine resistance against acids. ${ }^{18}$ Fluoride acts by chemically blocking fluid flow in the dentinal tubules by forming calcium-phos- 
phorous precipitates, calcium fluoride, and fluorapatite. Fluoride ions themselves do not occlude tubules; the source of desensitizing effects is the formation of precipitates. Basically, fluoride is known for its role in caries prevention that reduces the rate of demineralization, promotes remineralization of damaged tissue, and decreases acid production by interfering with oral bacteria in the tooth biofilm, by functioning as a biocide against Streptococcus mutans. Nevertheless, fluoride in combination with other ions may enhance the effectiveness of desensitising effects., ${ }^{9,21}$ Toothpastes may contain or induce the formation of calcium and phosphate and therefore contribute to intratubular mineralization. ${ }^{5,18,22}$ In addition, calcium phosphate is a promising agent in $\mathrm{DH}$ treatment. Calcium is important for remineralization in teeth restoration and is the primary component of hydroxyapatite. Apatite is a form of calcium phosphate used in dental materials, such as hydroxyapatite and fluorapatite. Precipitation of hydroxyapatite on an exposed dentine surface can occlude dentinal tubules. All in all, calcium phosphate can occlude dentinal tubules without inhibiting spontaneous remineralization of the tooth surface. ${ }^{15,21}$

Moreover, dentifrices can also contain strontium, stannous, and calcium phosphate. These elements can form physical barriers that occlude dentinal tubules. These mechanisms occur by precipitating insoluble metal compounds on the dentine surface. Stannous chloride has been proved efficient in occluding dentinal tubules. It should be noted that NovaMin ${ }^{\circledR}$ bioactive glass has been shown to be more effective than strontium chloride and placebo, even when exposed to citric acid and artificial saliva. ${ }^{8}$ Stannous fluoride can block tubules by forming SnF2 and CaF2, particles that can occlude the dentinal tubules. ${ }^{18}$

L Wang et al. compared FGM's Desensibilize Nano-P paste, Desensibilize Nano-P (associated with experimental home-care pastes), Colgate ${ }^{\circledR}$ Sensitive Pro-Relief ${ }^{\mathrm{TM}}$ professional paste (associated with home-care toothpaste), and Duraphat professional varnish. The results showed that although $\mathrm{NaF}$ varnish had a small effect on reducing $\mathrm{DH}$, the other treatments were all equally strong. Therefore, nanohydroxyapatite formulations and toothpastes with ProArgin technology are both good treatment options. ${ }^{23}$ Moreover, Yang et al., conducted a meta-analysis study that tested the tubule occlusion potency of arginine-containing toothpastes and showed that they had a superior therapeutic effect relative to the effects of other desensitizing products. ${ }^{24}$

Therefore, the ProArgin technology of Colgate ${ }^{\circledR}$ Sensitive Pro-Relief ${ }^{\mathrm{TM}}$ provides immediate relief of $\mathrm{DH}$ relative to the relief from KNO3 toothpaste and from a fluoride toothpaste. $^{25}$

Nevertheless, according to Rithesh et al., it was observed that in an in vitro comparison of toothpastes including nanohydroxyapatite, NovaMin, and ProArgin (Colgate ${ }^{\circledR}$ Sensitive
Pro-Relief $\left.{ }^{\mathrm{TM}}\right)$, all three desensitizing agents occluded dentinal tubules. However, there was a significant difference between their effectiveness. The percentage of tubule occlusion was $97.62 \%$ for nanohydroxyapatite formulation, $81.9 \%$ for NovaMin and $65.42 \%$ for ProArgin. The study found nanohydroxyapatite toothpaste was more effective for occluding dentinal tubules than were NovaMin and ProArgin. ${ }^{26}$

Tubule occlusion may also occur naturally involving normal remineralization processes by saliva and dentinal sclerosis through secondary dentine formation. Saliva has a protective function against tooth wear. The biofilm promotes remineralization that reduces mineral loss. ${ }^{9}$ Therefore, properties of the dentine can be improved to make it more resistant to mechanical and chemical attack. One method is to increase dentine surface resistance to wear caused by acid erosion and abrasion by increasing its mineral density. Another method is to fill the tubules with a mineral substance, such as calcium and phosphate toothpaste, that would increase acid resistance of dentine.,8

\section{Bioactive Glass-Based Toothpastes}

There are a number of desensitizing toothpastes available in the market, but among bioactive glass-based toothpastes, the options are limited. The best dentifrices to treat $\mathrm{DH}$, mentioned in studies regarding their efficacy, are Sensodyne ${ }^{\circledR}$ Repair \& Protect, which contains NovaMin $^{\circledast}$ (45S5 composition), and BioMinC ${ }^{\otimes}$ and BioMinF$^{\circledR}$ (based on calcium phospho-silicates). The 45S5 glass (NovaMin) reacts with saliva and releases calcium and phosphate ions that bind to collagen and form a hydroxyapatite layer. This layer can occlude the dentinal tubules and prevent $\mathrm{DH}^{26-29}$ However, BioMin, which is based on calcium phospho-silicates (bioactive glass), has a different composition from that on NovaMin. BioMin has a higher phosphate content, which speeds up apatite formation, lowers silica levels, and has smaller particles; therefore, it can better infiltrate tubules and is less abrasive to enamel. BioMinF also contains fluoride. Hence, when in contact with saliva, BioMinF releases calcium, phosphate, and fluoride ions that dissolve slowly, which creates long-lasting protection and forms fluorapatite. Fluorapatite is more resistant to acids and can promote remineralization. Most toothpaste contains soluble fluoride, which is easily washed away after a few hours. BioMin contains a polymer that binds calcium in the bioglass to calcium on the enamel and therefore, prevents the bioglass to be easily washed away. ${ }^{30,31}$

\section{Discussion}

It has become evident that bioactive glasses can occlude dentinal tubules so incorporating these glasses into toothpaste formulations may be beneficial for individuals who 
suffer from DH. ${ }^{19}$

Neuhaus et al. published a study in $2013^{32}$ that compared the effectiveness of a toothpaste containing $15 \%$ calcium sodium phosphosilicate with and without fluoride in a clinical study in subjects with $\mathrm{DH}$. The subjects brushed their teeth twice a day for 2 (timed) minutes during 14 days of testing. Immediately and 18 days after brushing, DH was assessed by both tactile and air blast stimuli. The results showed a significant reduction in sensitivity after 28 days of treatment for both toothpaste groups with or without fluoride, which suggests that any improvement in $\mathrm{DH}$ was independent of the presence of fluoride. Nevertheless, in another study by Lynch E. et al, toothpaste containing fluoride showed a significant percentage of tubular occlusion. ${ }^{30}$ Additionally, bioactive glass-based toothpastes containing have several benefits for tooth structure in addition to tubule occlusion.

In a novel laboratory study, ${ }^{16}$ the investigators synthesized a varnish containing potassium chloride $(\mathrm{KCl})$ and fluoridated hydroxyapatite (FHA). SEM images obtained from the paper showed that the dentinal tubules were occluded in the varnish FHA group. KCl-FHA varnish could release potassium ions and reduce hydraulic conductance of the dentine discs, so it could be a suitable option for the treatment of DH. FHA can occlude dentinal tubules over time; however, the samples were not subjected to an acidic challenge or other food and beverages that would have an impact in the clinical environment.

A further in vitro study ${ }^{6}$ by Gillam et al. reported that toothpaste formulations with different proportions of bioglass replacing the silica compounds (2.5\% and $7.5 \%)$ provided greater surface coverage than that of the original bioglass product. However, the study did not assess the composition of the particles deposited on the dentine surface or in the dentinal tubules. There was no reported determination on whether the deposit was an abrasive component e.g., silica or whether the deposit consisted of bioglass particles or a precipitation of calcium phosphate following ion exchange on the surface of bioglass.

Recent studies have made considerable progress in elucidating the effects of bioglass on tooth structure through in vitro evaluation of different chemical compositions of bioglass-containing toothpastes. ${ }^{15,21}$ For example, different outcomes have been reported when evaluating chloride-containing toothpastes. In vitro studies have demonstrated that a small crystalline deposit is precipitated onto the dentine surface, which can easily be rinsed away; however, other studies have reported positive effects of chloride-containing toothpaste on DH relief. ${ }^{15,21}$

Regarding comparisons of chlorine and fluoride toothpastes, there have been conflicting results reported in the literature. Several clinical studies have indicated that there were no differences in efficacy between the two products, and other studies, have indicated that there were differences in favor of chloride-containing toothpastes. This observation was also true when comparing in vitro studies alone, and in comparative studies using cross-sections of dentine where no tubular occlusion was observed following treatment with a SnF2-containing toothpaste. ${ }^{18}$

Moreover, a systematic review by Splieth \& Tachou ${ }^{14}$ presented an overview of clinical trials of calcium sodium phosphosilicate (CSPS) to treat DH. CSPS has been reported to provide $\mathrm{DH}$ reduction results superior to those for potassium nitrate-containing toothpastes. Investigators have suggested that when in contact with body fluids, CSPS reacts by forming a layer of hydroxyapatite that can occlude dentinal tubules. CSPS also has been reported to be more effective than negative controls. ${ }^{14}$

One final review that may be relevant in this discussion on the effectiveness of CSPS is a systematic review by Talioti \& Gillam. ${ }^{33}$ These investigators compared the evidence of OTC desensitizing products (e.g., calcium sodium phosphosilicate, amorphous calcium phosphate, nanohydroxyapatite, and tooth mousse toothpaste/gels) in reducing $\mathrm{DH}$. One of the problems reported by these investigators was that there was a lack of published studies directly comparing these four products. Furthermore, although there was evidence for the effectiveness of CSPS in occluding dentinal tubules no conclusion could be made regarding the clinical efficacies of the various desensitizing toothpastes compared in the review. This was partly because of the different study designs and methodologies used in the various studies and that there were relatively few randomized controlled trials available for analysis. ${ }^{33}$

Therefore, after analyzing several studies on the treatment of DH, we found that there are considerable discrepancies between studies which may be because of differences in processing of the dentine specimens, in the preparation of samples, in the modes of application of the dentifrice, and in manufacturing singularities. The findings of the present study promote the use of bioactive glass-based toothpastes for home application, because of its low cost, ease of application, and distinct evidence of its benefits in the published literature.

$\mathrm{DH}$ is a common clinical condition among most patients at present. The increase in cases is related to non-carious lesions, such as erosion, abrasion, abfraction, and attrition that can cause the symptoms of DH. For that reason, it is extremely important that professionals familiarize themselves with the condition and treatment options available in the market.

It is also important to treat the cause of the lesion that is causing DH. For instance, in cases of tooth pain caused by an abfraction lesion, instead of a composite restoration treatment, professionals should be able to identify what 
caused the abfraction that caused the $\mathrm{DH}$ and treat it correctly while always prioritizing minimally invasive dentistry.

\section{Conclusion}

The results of this review support the following conclusions: the materials available for $\mathrm{DH}$ treatment range from desensitizing mouthwashes and dentifrices to varnishes and restorative materials; toothpaste formulations containing bioactive glasses occlude the dentinal tubules and may be an effective treatment for DH; bioactive glass-based toothpastes are a great option for treating $\mathrm{DH}$ because of the ease of application and low cost relative to those of other in-office treatments and further research is required to determine the best approaches and increase patient quality of life.

\section{References}

1. Davari AR, Ataei E, Assarzadeh H. Dentin Hypersensitivity: Etiology, Diagnosis and Treatment. J Dent (Shiraz). 2013;14(3):136-45.

2. Hench LL. The story of Bioglass ${ }^{\oplus}$. J Mater Sci Mater Med. 2006;17(11):967-78.

3. Miglani S, Aggarwal V, Ahuja B. Dentin Hypersensitivity: Recent trends in management. J Conserv Dent. 2010;13(4):218-24.

4. Gillam DG. Dentine Hypersensitivity: Advances in Diagnosis, Management, and Treatment. Cham: Springer International Publishing; 2015.

5. Markowitz K, Pashely DH. Discovering new treatments for sensitive teeth: the long path from biology to therapy. J Oral Rehabil. 2008;35(4):300-15.

6. Gillam DG, Tang JY, Mordan NJ, Newman HN. The effects of a novel Bioglass ${ }^{*}$ dentifrice on dentine sensitivity: a scanning electron microscopy investigation. J Oral Rehabil. 2002;29(4):305-13.

7. West NX, Lussi A, Seong J, Hellwig E. Dentin hypersensitivity: pain mechanisms and aetiology of exposed cervical dentin. Clin Oral Investig. 2012;17(S1):9-19.

8. Pillai VP; Neelakantan P. Desensitizing Toothpastes for Treatment of Dentin Hypersensitivity. Int J PharmTech Res. 2013;5(4):1769-73.

9. Petersson LG. The role of fluoride in the preventive management of dentin hypersensitivity and root caries. Clin Oral Investig. 2012;17(S1):63-71.

10. Pashley DH. How can sensitive dentine become hypersensitive and can it be reversed? J Dent. 2013;41(04):49-55.

11. Prati C, Venturi L, Valdrè G, Mongiorgi R. Dentin Morphology and Permeability After Brushing with Different Toothpastes in the Presence and Absence of Smear Layer. J Periodontol. 2002;73(2):183-90.

12. Gillam DG. Current diagnosis of dentin hypersensitivity in the dental office: an overview. Clin Oral Investig. 2013;17(S1):21-9.

13. Zhu M, Li J, Chen B, Mei L, Yao L, Tian J, et al. The Effect of Calcium Sodium Phosphosilicate on Dentin Hypersensitivity: A Systematic Review and Meta-Analysis. PloS One. 2015;10(11):e0140176.

14. Splieth CH, Tachou A. Epidemiology of dentin hypersensitivity. Clin Oral Investig. 2012;17(S1):3-8.

15. Lochaiwatana Y, Poolthong S, Hirata I, Okazaki M, Swasdison S, Vongsavan $\mathrm{N}$. The synthesis and characterization of a novel potassium chloride-fluoridated hydroxyapatite varnish for treating dentin hypersensitivity. Dent Mater J. 2015;34(1):31-40

16. Acharya AB, Surve SM, Thakur SL. A clinical study of the effect of calcium sodium phosphosilicate on dentin hypersensitivity. J Clin Exp Dent. 2013;5(1):18-22.

17. Arnold WH, Prange M, Naumova EA. Effectiveness of various toothpastes on dentine tubule occlusion. J Dent. 2015;43(4):440-9.

18. Maldupa I, Brinkmane A, Rendniece I, Mihailova A. Evidence based toothpaste classification, according to certain characteristics of their chemical composition. Stomatologija, Baltic Dental and Maxillofacial Journal. 2012;14(1):12-22.

19. Cruz ACC, Da Silva JCZ, Pilatti GL, Santos FA. Use of Bioactive Glasses as Bone
Graft Substitutes: A Review of Literature. Revista de Odontologia da Universidade Cidade de São Paulo. 2006;18(3:)287-95.

20. Khoroushi M; Keshani F. A review of glass-ionomers: From conventional glass-ionomer to bioactive glass-ionomer. Dent Res J (Isfahan) 2013;10(4):411-20.

21. Davis HB, Gwinner F, Mitchell JC, Ferracane JL. Ion release from, and fluoride recharge of a composite with a fluoride-containing bioactive glass. Dent Mater. 2014;30(10):1187-94.

22. Wang Z, Sa Y, Sauro S, Chen H, Xing W, Ma X, et al. Effect of desensitising toothpastes on dentinal tubule occlusion: A dentine permeability measurement and SEM in vitro study. J Dent. 2010;38(5):400-10.

23. Wang L, Magalhães AC, Francisconi-Dos-Rios LF, Calabria MP, Araújo D, Buzalaf M, et al. Treatment of Dentin Hypersensitivity Using Nano-Hydroxyapatite Pastes: A Randomized Three-Month Clinical Trial. Oper Dent. 2016;41(4):93-101.

24. Yang Z, Wang F, Lu K, Li Y, Zhou Z. Arginine-containing desensitizing toothpaste for the treatment of dentin hypersensitivity: a meta-analysis. Clinical, Cosmetic and Investigational Dentistry. 2016;2016(8):1-14.

25. Dentine Hypersensitivity - Colgate-sensitive-pro-relief. [Internet]. [Cited in 2017 Feb 10] Available at :

http://www.colgateprofesional.com.ar/LeadershipAR/ProfessionalEducation/Articles/Resources/pdf/CSRP_Study_Ayad_instant.pdf

26. Kulal R, Jayanti I, Sambashivaiah S, Bilchodmath S. An In-vitro Comparison of Nano Hydroxyapatite, Novamin and Proargin Desensitizing Toothpastes - A SEM Study. J Clin Diagn Res. 2016;10(10):ZC51-ZC54.

27. Amaechi, B. Mathews SM, Ramalingam K, Mensinkai PK. Evaluation of nanohydroxyapatite-containing toothpaste for occluding dentin tubules. Am J Dent. 2015;28(1):33-9.

28. Acharya AB, Surve SM, Thakur SL. A clinical study of the effect of calcium sodium phosphosilicate on dentin hypersensitivity. J Clin Exp Dent. 2013;5(1):18-22.

29. Majji P, Murthy KR. Clinical efficacy of four interventions in the reduction of dentinal hypersensitivity: A 2-month study. Indian J Dent Res. 2016;27(5):477-82.

30. Lynch E, Brauer DS, Karpukhina N, Gillam DG, Hill RG. Multi-component bioactive glasses of varying fluoride content for treating dentin hypersensitivity. Dent Mater. 2012;28(2):168-78.

31. Here Comes The Science Bit. [Internet]. [Cited in 2017 Feb 10] Available at: $<$ http:// www.biomin.co.uk/wp-content/uploads/2016/05/DM-May-2-Biomin-F.pdf>.

32. Neuhaus KW, Milleman JL, Milleman KR, Mongiello KA, Simonton TC, Clark

$\mathrm{CE}$, et al. Effectiveness of a calcium sodium phosphosilicate containing prophylaxis paste in reducing dentine hypersensitivity immediately and 4 weeks after a single application: a double-blind randomized controlled trial. J Clin Periodontol. 2013;40(4):349-57.

33. Talioti E, Hill R, Gillam DG. The Efficacy of Selected Desensitizing OTC Products: A Systematic Review. ISRN Dent. 2014;2014:865761.

\section{Mini Curriculum and Author's Contribution}

1. Luiza Pereira Dias da Cruz - DDS. Contribution: writing, editing, and final approval.

2. Inger Teixeira de Campos Tuñas - DDS and PhD. Contribution: writing, review, and final approval 